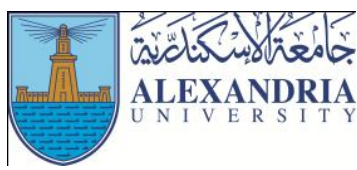

\author{
Journal of Applied Sports Science \\ June 2016, Volume 6, No. 2 \\ www.jass.alexu.edu.eg
}

\title{
Effect of Two Programs (Walking vs. Aquatic) with Weights on OPG in Adult Women
}

\author{
Dina Ali Mohamed Said ${ }^{1}$, Shaimaa Mohamed Naguib ${ }^{2}$ \\ ${ }^{1}$ Ass. Professor at the aquatic sports training department, Faculty of Physical education for girls, Helwan University, Egypt \\ ${ }^{2}$ Ass. Professor at track and field training department, Faculty of Physical education for girls, Helwan University, Egypt
}

\begin{abstract}
Aging is associated with physical impairment characterized by deterioration of bone mass and bone decalcification. physical activity helps prevent osteoporosis and Some forms of exercise may cause more profound effects on bone than others. Aim of work was to examine the acute response of bone markers post-exercise to pre-exercise and chronic response (adaptation) to training program for two separate groups of adult females subjected to either walking or aquatic training programs with weights. 20 adult sedentary females participated in either aquatic or walking training programs for three months, subdivided and subjected to a graded training program for three months in a legal basin or field track. There were significant variations in all physical parameters after program compared to that obtained before program in either both groups. Biochemical parameters of both bone synthesis and resorption parameters were elevated after effort in both groups (TRAP b5 and CICP). Osteoprotegerin was elevated in the resistance group only after the program after effort but not before program. It is concluded that a combination of regular recreational walking and/or swimming (both at the same intensity) are important to improve strength and flexibility and most of bone and muscle preservative criteria for most people.
\end{abstract}

Keywords: $O P G$, Old age, bone, aquatic exercise, walking program, Females.

\section{Introduction}

A ging is associated with several anatomical and physiological changes leading to physical impairment characterized by increased disability, frailty and falls due to the gradual deterioration of bone mass, gradual bone decalcification (osteopenia) and loss of muscle mass (sarcopenia). Women develop less peak bone and muscle mass than men and are more susceptible to the functional limitations that accompany the age-associated decay of bone health and muscle function (Pernambuco et al., 2013).

Physical activity (PA) has been proven to enhance muscle strength, balance, and quality of life (QoL); in addition to maintaining functional autonomy and lowering the risk of falls and fall-related injuries (Borba-Pinheiro et al., 2010). Young and Dinan (2005) postulated that physical activity helps prevent osteoporosis, hypertension, ischaemic heart disease, stroke, anxiety, depression, Disability, age related weakness, Immobility and Isolation.

The extracellular matrix of skeletal muscle, tendon, ligament, and bone is important for maintaining tissue structure and vital for transmission of force during muscular contraction. Since collagen is the major protein in the extracellular matrix of the musculoskeletal tissue, it is important to tissue function. Knowledge of the physiological regulation of the amount and turnover of collagen in human beings is poor (Babraj et al., 2005). Haus et al. (2007) hypothized that a critical component in the transfer of force from the contractile units of the muscle out to the tendon and subsequent bone is the connective tissue scaffold that surrounds individual muscle fibers, muscle bundles, and the whole muscle (Huijing, 1999; Kjaer , 2004). This skeletal muscle connective tissue network is primarily composed of collagen fibers and biochemical linkages within and between these fibers, which provide strength and stability.

Some forms of exercise may cause more profound effects on bone than others. This is due to several factors that are manifested in the form of exercise, such as the mechanical load of the exercise, intensity, and duration of exercise. High load exercise, such as weight bearing and plyometric exercises, lead to an increase in BMD at sites affected by strains and provide more osteogenic effects (Maimoun and Sultan, 2011). On the other hand, endurance and low-impact exercises (eg cycling) have been shown to have no beneficial or even deleterious effects on bone density (Guillemant et al., 2004). 
Walking involves all major muscle groups of the body and provides cardiorespiratory conditioning. Cardiorespiratory fitness assists to increase the proportion of high-density lipoprotein, strengthens the heart muscle and makes it more efficient, increases the capillary network of the body, improves circulation, stimulates bone growth, and aids in controlling body weight. Walking one mile requires the same energy as running one mile and the injury rate is less for walking. Therefore walking is safer for obese persons who are at a higher risk of joint injuries (Dallas, 1996).

Aquatic exercise is widely practiced by elderly women. However, such exercise does not induce significant osteogenic stimuli, due to the diminished impact on bones provoked by low gravitational action in the water environment (Tormen, 2007). On the other hand, Arnold et al. (2008) reported that aquatic exercise may contribute toward improving other risk variables for osteoporosis including strength, balance, QoL and functional autonomy.

During exercise activities (either walking or aquatic), resistance is added to movement in order to make muscles work harder and, over time, become stronger. The most common strength training methods include using weight machines, working with free weights, or doing exercises that use body weight (push-ups, for example). Although these resistance exercises focus on increasing muscle mass, they also put stress on bones and have bone-building capacity. (Zakia, 2004 \& Nariman and Abdel Aziz, 2014)

Osteoprotegrin (OPG) has an osteo-protective role in humans, protecting bones from excessive bone resorption. Experiments have shown that mechanical stimulation can inhibit osteoclast formation and activity by changing the OPG levels in favour of BMD. OPG is the soluble factor in bone that decreases bone resorption by binding to receptor activator of the nuclear factor-kB (RANK) on the osteoclast, its effect on skeletal muscle through paracrine and/or endocrine action. Relationship between the osteoprotegerin (OPG)/ (RANK)/RANK ligand (RANKL) system is the regulator of osteocyte/osteoclast activities leading to skeletal health due to its critical role in bone remodeling (Boyce and Xing, 2008).

However, the association between serum OPG and the incidence of bone fractures and BMD has been inconsistent (Marques et al., 2011). Type
I collagen is known to adapt to physical activity, and biomarkers of collagen turnover indicate that synthesis can be influenced by an intense exercise, but the exact time pattern of these changes are largely undescribed.

Although many studies used X-ray as the gold standard assessment for determining BMD, it has many disadvantages as it poses radiation risk to the individual and lacks a sensitive measure for the short-term effects of exercise on bone. Thus, a more practical method of assessing bone health utilizing biochemical factors such as bone formation and resorption markers (Banfi et al., 2012). These markers provide evidence of bone turnover and also a prediction of the cellular activities of osteoblasts and osteoclasts in bone formation and resorption.

\section{Aim of Work}

The overall objective of the present study, which included two measures (before and after) training program, was to examine the acute response of bone markers post-exercise to pre-exercise and chronic response (adaptation) to training program for two separate groups of adult females subjected to either walking or aquatic training programs with weights. Plasma osteoprotegerin and $\mathrm{C}$ terminal of type I procollagen (CICP) (marker for bone formation), enzyme serum band 5 tartarate resistant acid phosphatase (TRAP 5b) (marker of bone resorption).

The second aim is to generate new hypotheses introducing the structural basis for training exercise program-induced improvements in bone strength, as well as the characteristics of loading that have been shown to be best associated with structural improvements in bone either walking or aquatic.

\section{Material and Methods}

\section{Subjects}

20 adult sedentary females participated in either aquatic or walking training programs for three months in Maadi sport and yacht club, Maadi, Cairo from $7 / 3 / 2015$ to $27 / 5 / 2015$. The subjects voluntarily provided written informed consent before participating. There was no clinical or analytical evidence for any disease among the participants. Participants aged 54.21 \pm 4.07 years, height $167.21 \pm 5.44 \mathrm{~cm}$. and weight at the start of the program $83.01 \pm 14.67 \mathrm{k}$. They were subdivided and subjected to a graded training program for three months in a legal basin or field track.

\section{Study design:}

The participants underwent three months of moderate to intensive exercise units in every training session. Each unit consisted of 10 min jogging and stretching exercises as a warm-up followed by walking or swimming with weights for 45 mins., then 5 mins. As recovery. The training program was summarized in table (1). 
Training program graded through three months

\begin{tabular}{|c|c|c|c|c|c|}
\hline Month & \multicolumn{5}{|c|}{ Training session } \\
\hline & Sessions & intensity & Warm up & (Walking or swimming) with weights & recovery \\
\hline 1st & 12 & $40-60 \%$ & $10 \mathrm{~min}$ & $45 \mathrm{~min}$ & $5 \mathrm{~min}$ \\
\hline 2nd. & 12 & $60-70 \%$ & $10 \mathrm{~min}$ & $45 \mathrm{~min}$ & $5 \mathrm{~min}$ \\
\hline 3rd. & 12 & $70-80 \%$ & $10 \mathrm{~min}$ & $45 \mathrm{~min}$ & $5 \mathrm{~min}$ \\
\hline
\end{tabular}

\section{Physical studies:}

Physical parameters were measured for Right fist strength, Left fist strength, legs lift strength, Back lift strength and Abdomen lift strength by using dynamometer. Measurements were recorded before and after the training program.

\section{Blood analysis:}

Blood samples at rest were collected under aseptic conditions from the anti-cubital vein. Post-exercise blood sampling was performed immediately after the exercise unit. Venous samples for the measurement of plasma parameters were collected in tubes containing EDTA in the morning after fasting, after resting for at least 30 minutes and immediately after exercise, centrifuged and kept at $-20 \mathrm{C}^{\mathrm{o}}$ until tested. osteoprotegerin and $\mathrm{C}$ terminal type I procollagen beside TRAP $5 \mathrm{~b}$ levels were determined using a commercial ELISA kits provided by Teco medical group, Germany.

\section{Statistical analysis:}

All data are reported as mean \pm standard deviation (SD). The SPSS 17 software program was used to execute all statistical analyses. The paired sample t-test was used to compare the means of each group before and after the training program. All of the data were correlated to exercise. The level of significance was set at $\mathrm{p} \leq 0.05$.

\section{Results}

Physical results obtained before program revealed no significant variation in all parameters between the two groups. Table (2) represents results of these physical parameters. On the other hand, there is significant variation in all parameters after program compared to that obtained before program in either both groups. Regarding differences between the two groups, there were significant differences in Right fist strength, Left fist strength and legs lift strength but not between Back lift strength and Abdomen lift

strength.

Table (2)

Difference between results ( Mean \pm SD) obtained for physical parameters

\begin{tabular}{|c|c|c|c|c|c|}
\hline \multicolumn{6}{|c|}{ Walking with weights group } \\
\hline & Pre-program & Post-program & $\mathrm{t}$ & $\mathrm{p}$ & Significance \\
\hline Right fist strength & $9.51 \pm 2.41$ & $20.53 \pm 3.71$ & -14.580 & .000 & $\mathrm{~S}$ \\
\hline Left fist strength & $8.94 \pm 1.89$ & $19.46 \pm 3.62$ & -11.501 & .000 & $\mathrm{~S}$ \\
\hline legs lift strength & $27.8 \pm 4.57$ & $37.53 \pm 4.42$ & -8.538 & .000 & $\mathrm{~S}$ \\
\hline Back lift strength & $27 \pm 5.59$ & $46.8 \pm 7.30$ & -7.175 & .000 & $\mathrm{~S}$ \\
\hline Abdomen lift strength & $1.73 \pm 0.56$ & $5.79 \pm 2.27$ & -6.843 & .000 & $\mathrm{~S}$ \\
\hline \multicolumn{6}{|c|}{ Aquatic with weights group } \\
\hline & Pre-program & Post-program & $\mathrm{t}$ & $\mathrm{p}$ & Significance \\
\hline Right fist strength & $8.94 \pm 1.54$ & $31.6 \pm 4.17$ & -18.925 & .000 & $\mathrm{~S}$ \\
\hline Left fist strength & $8.83 \pm 1.97$ & $28.13 \pm 5.09$ & -13.180 & .000 & $\mathrm{~S}$ \\
\hline legs lift strength & $26.26 \pm 4.63$ & $40.6 \pm 5.79$ & -8.798 & .000 & $\mathrm{~S}$ \\
\hline Back lift strength & $48.53 \pm 4.95$ & $36.06 \pm 4.01$ & -8.982 & .000 & $\mathrm{~S}$ \\
\hline Abdomen lift strength & $1.93 \pm 0.53$ & $5.5 \pm 1.11$ & -6.714 & .000 & $\mathrm{~S}$ \\
\hline \multicolumn{6}{|c|}{ Aquatic vs. walking pre-program } \\
\hline & Walking & Aquatic & $\mathrm{t}$ & $\mathrm{p}$ & Significance \\
\hline Right fist strength & $9.51 \pm 2.41$ & $8.94 \pm 1.54$ & 1.587 & .135 & NS \\
\hline Left fist strength & $8.94 \pm 1.89$ & $8.83 \pm 1.97$ & .455 & .656 & NS \\
\hline
\end{tabular}


Dina Ali, Shaimaa Naguib

\begin{tabular}{|c|c|c|c|c|c|}
\hline legs lift strength & $27.8 \pm 4.57$ & $26.26 \pm 4.63$ & .978 & .344 & NS \\
\hline Back lift strength & $27 \pm 5.59$ & $48.53 \pm 4.95$ & -1.447 & .170 & NS \\
\hline Abdomen lift strength & $1.73 \pm 0.56$ & $1.93 \pm 0.53$ & -2.103 & .054 & NS \\
\hline \multicolumn{6}{|c|}{ Aquatic vs. walking post-program } \\
\hline & Aquatic & Walking & $\mathrm{t}$ & $\mathrm{p}$ & Significance \\
\hline Right fist strength & $20.53 \pm 3.71$ & $31.6 \pm 4.17$ & -7.950 & .000 & $\mathrm{~S}$ \\
\hline Left fist strength & $19.46 \pm 3.62$ & $28.13 \pm 5.09$ & -6.223 & .000 & $\mathrm{~S}$ \\
\hline legs lift strength & $37.53 \pm 4.42$ & $40.6 \pm 5.79$ & -2.817 & .014 & $\mathrm{~S}$ \\
\hline Back lift strength & $46.8 \pm 7.30$ & $36.06 \pm 4.01$ & .953 & .321 & NS \\
\hline Abdomen lift strength & $5.79 \pm 2.27$ & $5.5 \pm 1.11$ & 650 & .596 & NS \\
\hline
\end{tabular}

Three amazing results were obtained for the biochemical parameters of both bone synthesis and resorption parameters were elevated after effort in both groups (TRAP b5 and CICP) indicating acute response for them either before or after the program (Table 3). TRAP b5 only preserved these results for chronic response but not CICP which became stable after the program without differences in rest or after effort in both groups. Osteoprotegerin (OPG) results were more amazing and profound. It was elevated in the resistance group only after the program after effort but not before program at all. That is to say aquatic exercises didn't affect OPG while resistance exercises affect the chronic elevation after effort.

Table (3)

Difference between results ( Mean \pm SD) obtained for biochemical parameters

\begin{tabular}{|c|c|c|c|c|c|}
\hline \multicolumn{6}{|c|}{ Walking group (pre vs. post) program } \\
\hline \multicolumn{6}{|c|}{ Rest } \\
\hline & Pre & Post & $\mathrm{t}$ & $\mathrm{p}$ & Significance \\
\hline TRAP b5 & $2.65 \pm 0.48$ & $3.47 \pm 0.41$ & -6.441 & .000 & $S$ \\
\hline $\mathrm{CICP}$ & $99.8 \pm 17.76$ & $116.4 \pm 13.32$ & -2.826 & .013 & $\mathrm{~S}$ \\
\hline OPG & $5.03 \pm 1.05$ & $5.70 \pm 0.76$ & -2.205 & .045 & NS \\
\hline \multicolumn{6}{|c|}{ Effort } \\
\hline TRAP b5 & $3.48 \pm 0.56$ & $3.94 \pm 0.39$ & -3.231 & .006 & $S$ \\
\hline CICP & $134.2 \pm 13.44$ & $145.2 \pm 14.94$ & -2.713 & .017 & $S$ \\
\hline OPG & $5.25 \pm 0.88$ & $6.54 \pm 0.80$ & -4.302 & .001 & $S$ \\
\hline \multicolumn{6}{|c|}{ Aquatic group (pre vs. post) program } \\
\hline \multicolumn{6}{|c|}{ Rest } \\
\hline & Pre & Post & $\mathrm{t}$ & $\mathrm{p}$ & Significance \\
\hline TRAP b5 & $2.65 \pm 0.48$ & $3.48 \pm 0.56$ & -3.202 & .006 & $S$ \\
\hline CICP & $99.8 \pm 17.76$ & $134.2 \pm 13.44$ & -1.321 & .208 & NS \\
\hline OPG & $5.03 \pm 1.05$ & $5.25 \pm 0.88$ & -.455 & .656 & NS \\
\hline \multicolumn{6}{|c|}{ Effort } \\
\hline TRAP b5 & $3.47 \pm 0.41$ & $3.94 \pm 0.39$ & -3.986 & .001 & $S$ \\
\hline CICP & $116.4 \pm 13.32$ & $145.2 \pm 14.94$ & -1.321 & .208 & NS \\
\hline OPG & $5.70 \pm 0.76$ & $6.54 \pm 0.80$ & -2.680 & .018 & $S$ \\
\hline
\end{tabular}


Table (3b)

Difference between results ( Mean \pm SD) obtained for biochemical parameters

\begin{tabular}{|c|c|c|c|c|c|}
\hline \multicolumn{6}{|c|}{ Walking group (Rest vs. effort) } \\
\hline \multicolumn{6}{|c|}{ Pre-program } \\
\hline & Rest & Effort & $\mathrm{t}$ & $\mathrm{p}$ & Significance \\
\hline TRAP b5 & $2.65 \pm 0.48$ & $3.48 \pm 0.56$ & -4.588 & .000 & $\mathrm{~S}$ \\
\hline CICP & $99.8 \pm 17.76$ & $134.2 \pm 13.44$ & -5.551 & .000 & $\mathrm{~S}$ \\
\hline OPG & $5.03 \pm 1.05$ & $5.25 \pm 0.88$ & -1.943 & .072 & NS \\
\hline \multicolumn{6}{|c|}{ Post-program } \\
\hline TRAP b5 & $3.47 \pm 0.41$ & $3.94 \pm 0.39$ & -2.789 & .015 & $\mathrm{~S}$ \\
\hline CICP & $116.4 \pm 13.32$ & $145.2 \pm 14.94$ & -6.396 & .000 & $\mathrm{~S}$ \\
\hline OPG & $5.70 \pm 0.76$ & $6.54 \pm 0.80$ & -3.866 & .002 & $\mathrm{~S}$ \\
\hline \multicolumn{6}{|c|}{ Aquatic group (Rest vs. effort) } \\
\hline \multicolumn{6}{|c|}{ Pre-program } \\
\hline & Rest & Effort & $\mathrm{t}$ & $\mathrm{p}$ & Significance \\
\hline TRAP b5 & $2.71 \pm 0.47$ & $3.53 \pm 0.44$ & -4.581 & .000 & $\mathrm{~S}$ \\
\hline CICP & $92.13 \pm 13.12$ & $139.66 \pm 11.61$ & -13.443 & .000 & $\mathrm{~S}$ \\
\hline OPG & $5.10 \pm 0.73$ & $4.97 \pm 0.69$ & .444 & .663 & NS \\
\hline & \multicolumn{2}{|c|}{ Post-program } & & & \\
\hline TRAP b5 & $3.05 \pm 0.35$ & $4.05 \pm 0.46$ & -7.662 & .000 & $S$ \\
\hline CICP & $98.33 \pm 15.94$ & $130.13 \pm 14.21$ & -5.961 & .000 & $S$ \\
\hline OPG & $5.23 \pm 0.84$ & $5.65 \pm 0.65$ & -1.440 & .172 & NS \\
\hline
\end{tabular}

More profound results were obtained when aquatic group was compared to walking group as both resorption and deposition markers were elevated after effort before program indicating active bone metabolism in both directions. After the program the most amazing result were the insignificant differences in resorption results after effort which indicating stability of bone formation without more resorption (Table 4). Osteoprotegrin was found to be elevated only after effort in post program samples indicating adapted response to program with increasing osteocytes activities against decreased osteoclasts activities.

Table (4)

Difference between walking vs. aquatic results (Mean \pm SD) obtained for biochemical parameters

\begin{tabular}{|c|c|c|c|c|c|}
\hline \multicolumn{6}{|c|}{ Walking vs aquatic group (Rest) } \\
\hline \multicolumn{6}{|c|}{ Pre-program } \\
\hline & Walking & Aquatic & $\mathrm{t}$ & $\mathrm{p}$ & Significance \\
\hline TRAP b5 & $2.65 \pm 0.48$ & $2.71 \pm 0.47$ & -.342 & .737 & NS \\
\hline CICP & $99.8 \pm 17.76$ & $92.13 \pm 13.12$ & 1.959 & .070 & NS \\
\hline OPG & $5.03 \pm 1.05$ & $5.10 \pm 0.73$ & -.210 & .836 & NS \\
\hline \multicolumn{6}{|c|}{ Post-program } \\
\hline & Walking & Aquatic & $\mathrm{t}$ & $\mathrm{p}$ & Significance \\
\hline TRAP b5 & $3.47 \pm 0.41$ & $3.05 \pm 0.35$ & 2.746 & .016 & $S$ \\
\hline CICP & $116.4 \pm 13.32$ & $98.33 \pm 15.94$ & 3.341 & .005 & $S$ \\
\hline OPG & $5.70 \pm 0.76$ & $5.23 \pm 0.84$ & 1.570 & .139 & NS \\
\hline \multicolumn{6}{|c|}{ Walking vs aquatic group (Effort) } \\
\hline \multicolumn{6}{|c|}{ Pre-program } \\
\hline & Walking & Aquatic & $\mathrm{t}$ & $\mathrm{p}$ & Significance \\
\hline TRAP b5 & $3.48 \pm 0.56$ & $3.53 \pm 0.44$ & -.271 & .790 & NS \\
\hline
\end{tabular}




\begin{tabular}{|c|c|c|c|c|c|}
\hline CICP & $134.2 \pm 13.44$ & $139.66 \pm 11.61$ & -1.090 & .294 & NS \\
\hline OPG & $5.25 \pm 0.88$ & $4.97 \pm 0.69$ & .879 & .394 & NS \\
\hline \multicolumn{7}{|c|}{ Post-program } & t & $\mathrm{p}$ & Significance \\
\hline & Walking & Aquatic & -.606 & .554 & NS \\
\hline TRAP b5 & $3.94 \pm 0.39$ & $4.05 \pm 0.46$ & 3.773 & .002 & $\mathrm{~S}$ \\
\hline CICP & $145.2 \pm 14.94$ & $130.13 \pm 14.21$ & 3.638 & .003 & $\mathrm{~S}$ \\
\hline OPG & $6.54 \pm 0.80$ & $5.65 \pm 0.65$ & T
\end{tabular}

\section{Discussion}

The observation made in the present study was to investigate bone response and metabolism before and after training exercise program either aquatic or walking with weights. Both programs had increased physical fitness of investigated subjects. Since many previous results showed negative improvement in aquatic trainings in general, this study had proved positive progress. This may be due to weights tied to legs and hands of subjects.

Mohr et al. (2015) demonstrated improvements in lower limb bone mass and bone strength after 15 weeks of soccer training in sedentary middle-aged women, whereas no effect was observed with swim training. Prospective data from a large and diverse cohort of adult women indicate that walking exercise is associated with reductions in the risk of cardiovascular events. Moreover, prolonged time spent sitting predicted increased risk. These findings support to current guidelines that endorse moderate intensity activity, including walking (Manson et al., 2002). Our results indicate that moderate-intensity exercise confers substantial health benefits for old women regardless aquatic or walking.

Exercising in water also can significantly improve cardiorespiratory fitness, muscular strength, body composition, blood lipids, agility, and flexibility in older adults. Moreover, it may provide additional benefits by reducing the incidence of falls and injuries that occur while performing exercise, or while performing activities of daily living. Therefore, water-based exercise is a beneficial mode of exercise for older adults and can be safely used as part of a well-rounded exercise program (Takeshima et al., 2002). The proposed aquatic exercise program was efficient in attenuating bone resorption raise and enhancing bone formation, which prevented the participants from reducing the femoral trochanter BMD (Moreira et al., 2014). Results showed an augment in bone formation marker (P1NP) only in the walking group.
TRAP b5 was evaluated for bone resorption, CICP for bone deposition and OPG for bone cellular metabolism (osteoclast vs. osteoblast) activities in response to the training program. There were significant differences observed at rest (pre-exercise) compared to after exercise effort (post-exercise) in all results obtained except for OPG (Table 3) compared to themselves, which found to be elevated after effort after the training program.

A combination of regular recreational walking and/or swimming (both at the same intensity), preferably combined with specific exercises to improve strength and flexibility, will meet most of these criteria for most people. Many older people also welcome the opportunity to participate in group exercise.

It is established that mechanical force through gravity or muscle pull is a major factor in maintaining or increasing bone mass and bone strength. Evidence showed that exercise can contribute to the prevention of osteoporosis by increasing bone mineral density (BMD), reducing age-related bone loss, or restoring bone already lost in the elderly.

Weight bearing physical activity (or resistance exercise) and increased mechanical loading had beneficial effects on bone mineralization and development in children and adolescents, particularly during periods of rapid bone growth (Hind and Burrows, 2007). Exercises that affect skeleton including aerobics, football and gymnastics; resistance training; plyometrics; circuit training; jumping exercises or a combination of exercises. Moreover, many studies have revealed that the progressive effects of exercises on increasing bone strength, reducing fracture risk and a given peak BMD depend on the optimal intensity, frequency and duration of such exercise and the location of bone. Karlsson and Krustrup (2010) found that exercise by playing competitive national-league soccer at more than 6 hours per week did not appear to confer any added BMD benefit to men. Whereas daily hopping exercises over 6 months could increase femoral neck BMD in premenopausal women but less frequent exercise had no significant effect (Bailey and Brooke- Wavell, 2010).

Study in old women with low bone mass revealed that the agility training significantly increased cortical bone density at the tibial shaft, whilst the resistance training significantly increased cortical bone density at the radial shaft (Liu- Ambrose et al., 2004). 
Although both resistance exercise and aerobic exercise demonstrated protecting against the functional balance control that was strongly related to fall risk in Caucasian old women with 8 months (3 times per week) of resistance exercise, the resistance exercise was more effective than aerobic exercise for inducing favorable changes in BMD and muscle strength (Marques et al., 2011).

Furthermore, dynamic loads were indicated to be determinants of Poly bone mass (PBM). Results showed that external joint moments (during walking) enhanced predictability of proximal femoral BMD and bone mass content (BMC) when compared to that of height and/or body mass with as much as $40 \%$ of the BMD at the proximal hip by dynamic loading information.

In addition, participation in long-term (8 weeks) physical training program resulted in increased biomarkers of bone formation without substantial alterations in the markers of bone resorption (in young healthy women). Whereas, strength training over 9 months (a 50-min strength training, twice weekly for 15 weeks) did not lead to significantly greater change in total body or regional BMD in women (Sherk et al., 2013).

Besides protecting against osteoporosis, preventing falls and reducing fracture risk, the recent population based study in adult men and women also reported that changes in physical activity were associated with changes in both BMD (increases) and in BMI (decreases), and increases in physical activity had the potential to decrease the obesity epidemic without increasing the risk of bone loss (Langsetmo et al., 2012).

Plasma osteoptotegrin was first to be estimated as a marker for bone response to a training program. Results were significant in after program compared to before program. These results may explain under the hypothesis that it affects bone mass rather than releasing in lymphatic system. In addition, basic and clinical studies have established a consistent relationship between the osteoprotegerin (OPG)/receptor activator of the nuclear factor-kB (RANK)/RANK ligand (RANKL) system whereas skeletal health is due to its critical role in bone remodeling. OPG has an osteo-protective role in humans, protecting bones from excessive bone resorption via binding to RANKL and preventing it from binding to RANK (Boyce and Xing, 2008). In vitro and in vivo experiments have shown that mechanical stimulation can inhibit osteoclast formation and activity by changing the OPG/RANKL ratio in favour of OPG (Saunders et al., 2006).

We also hypothesized that exercise would modify the acute bone response resulted in post-exercise compared to pre-exercise in all investigated parameters (Table 3) due to weights added to exercise regimen. These results seem to be related to the timing of the measurement of bone turnover markers relative to the last exercise bout or the diurnal variation of bone resorption markers (Henriksen et al., 2009) rather than the training program effect.

Bonewald and Johnson (2008) hypothized that osteocytes appear to be reflect the strain signals and distribute them throughout the whole bone to regulate bone (re)modeling. Recently it has been shown that deletion of osteocytes results in bone loss and bone with deleted osteocytes does not respond to load. These studies show that osteocytes are necessary to maintain bone mass in response to normal load, but in the absence of load, they send signals of resorption. Hence, as revealed in the present study, the net result of training program lead to bone deposition with elevated CICP as bone formation marker.

Rogers et al. (2011) studied the acute response of bone formation and resorption markers including tartrate-resistant acid phosphatase 5b (TRAP 5b), COOH terminal telopeptide of type I collagen (CTX), to a single bout of resistance exercise or polymetric exercise. In general, they observed cumulative decreases in TRAP5b during the $2 \mathrm{~h}$ following exercise while CTX remained unchanged. Their results suggest that the timing of the measurement is important for detection of exerciseassociated changes in bone turnover markers, as the markers returned to pre-exercise values within $2 \mathrm{~h}$ of exercise.

Virtanen et al. (1993) reported that a single bout of heavy concentric exercise causes protein leakage from muscles and probably from the collagen-synthesizing cells of the connective tissue, which may be accompanied by an initial decrease and a subsequent increase in type I collagen production. The activation of type I collagen production seems to depend on the strain and damage of the musculoskeletal system.

\section{Conclusion}

It is concluded that training programs with weights have profound effects on both physical and chemical structure of bone in adult women. Walking is better for some muscle strength, bone mineralization and OPG than aquatic although aquatic has also profound effects.

It is important for adults to participate in any physical activity to keep bone metabolism and prevent osteoporosis or osteomalecia. Bone minerals requires exercise training to load positive bone metabolism revisable to negative obtained after menopause.

\section{References}


1- Arnold, C. M., Schachter, B. A., Harrison, C. L., \& Olszynski, E.L.W.P. (2008). A randomized clinical trial of aquatic and land exercise to improve balance, function and quality of life in older women with osteoporosis. Physiotherapy Canada, 60, 296306.

2- Babraj JA, Cuthbertson DJ, Smith K, Langberg H, Miller B, Krogsgaard MR, Kjaer M, Rennie MJ. Collagen synthesis in human musculoskeletal tissues and skin. Am J Physiol Endocrinol Metab 289: E864-E869, 2005.

3- Bailey CA, Brooke-Wavell K: Optimum Frequency of Exercise for Bone Health: Randomised Controlled Trial of a High-Impact Unilateral Intervention. Bone 2010, 46:1043-1049.

4- Banfi, G. , Colombini, A. , Lombardi, G. , \& Lubkowska, A. (2012). Metabolic markers in sports medicine. Advances in Clinical Chemistry, 56, 1-54

5- Bonewald, L.F., Johnson, M.L. (2008). Osteocytes, mechanosensing and Wnt signaling. Bone, 42, 606-615.

6- Borba-Pinheiro, C. J., Carvalho, M.C.G.A., Silva, N. S. L., Drigo, A. J., Bezerra, J. C. P., \& Dantas, E. H. M. (2010). Bone density, balance and quality of life of postmenopausal women taking alendronate, participating in different physical activity programs. Therapeutic Advances in Musculoskeletal Disease, 2, 175-185.

7- Boyce, B.F., Xing, L., 2008. Functions of RANKL/RANK/OPG in bone modeling and remodeling.Arch. Biochem. Biophys. 473, 139146.

8- Dallas MI: Exercise Walking for Obesity Management in Older Adult White Women (M Sc.Thesis).Grand Valley State University, 1996

9- Guillemant, J. , Accarie, C. , Peres, G. , \& Guillemant, S. (2004). Acute effects of an oral calcium load on markers of bone metabolism during endurance cycling exercise in male athletes. Calcified Tissue International, 74(5), 407-14

10- Haus JM, Carrithers JA, Trappe SW, Trappe TA. Collagen, cross-linking, and advanced glycation end products in aging human skeletal muscle.J Appl Physiol 103: 2068-2076, 2007.

11- Hind K. and Burrows M. Weight-bearing exercise and bone mineral accrual in children and adolescents: a review of controlled trials. Bone. 2007; 40(1): 1427.

12- Henriksen, K., Neutzsky-Wulff, A.V., Bonewald, L.F., Karsdal, M.A. (2009). Local communication on and within bone controls bone remodeling. Bone, 44, 1026-1033.

13- Huijing PA. Muscle as a collagen fiber reinforced composite: a review of force transmission in muscle and whole limb.J Biomech 32: 329-345, 1999.

14- Karlsson MK, Krustrup P (2010) Recreational football training decreases risk factors for bone fractures in untrained premenopausal women. Scand J Med Sci Sports 20(Suppl 1):3139

15- Kjaer M. Role of extracellular matrix in adaptation of tendon and skeletal muscle to mechanical loading. Physiol Rev 84: 649698, 2004.

16- Langsetmo L, Hitchcock CL, Kingwell EJ, Davison KS, Berger C, Forsmo S, Zhou W, Kreiger N, Prior JC.: Physical Activity, Body Mass Index and Bone Mineral DensityAssociations in a Prospective Population-based Cohort of Women and Men: The Canadian Multicentre Osteoporosis Study (CaMos)Bone. 2012 January ; 50(1): 401-408.

17- Liu-Ambrose TY, Khan KM, Eng JJ. Both resistance and agility training increase cortical bone density in 75- to 85-yearold women with low bone mass: a 6-month randomized controlled trial.J. Clin. Densitom., 2004; 7 (4): 390-8

18- Manson J, Reenland Hg, Roix N, Tefanick A, Outon H, Berman L, Heps A, Ettinger A, Iscovick A .: Walking Compared With Vigorous Exercise For The Prevention Of Cardiovascular Events In Women .N Engl J Med 2002;347:716-25

19- Marques EA, Wanderley F, Machado L, Sousa F, Viana JL, Moreira-Gonçalves D, Moreira P, Mota J, Carvalho J.: Effects of resistance and aerobic exercise on physical function, bone mineral density, OPG and RANKL in older women.Experimental Gerontology 46 (2011) 524-532

20- Maimoun, L. , \& Sultan, C. (2011). Effects of physical activity on bone remodeling. Metabolism: Clinical and Experimental, 60(3), 373-88

21- Mohr M, Helge ME, Petersen LF, Lindenskov A, Weihe P, Mortensen J, J̈rgensen NR, Krustrup P.: Effects of soccer vs swim training on bone formation in sedentary middle-aged women.Eur J Appl Physiol., 421(15):3231-8

22- Nariman El Khateeb and Abdel Aziz El Nemr (2014): Sports traing : Theoritical basis and practical applicatios (Book).Arabic sports liberary (Pub),Cairo, Egypt. 2014

23- Moreira LD, Fronza FC, Santos RN, Zach PL, Kunii IS, Hayashi LF, Teixeira LR, Kruel LF and Castro ML: The benefits 
of a high-intensity aquatic exercise program (HydrOS) for bone metabolism and bone mass of postmenopausal women.Journal of Bone and Mineral Metabolism; 2014, 32 (4): 411-419

24- Pernambuco CS, Borba-Pinheiro CJ, de Souza Vale RG, Di Masi f, Monteiro PK, Dantas EH. (2013): Functional autonomy, bone mineral density (BMD) and serum osteocalcin levels in older female participants of an aquatic exercise program (AAG). Archives of Gerontology and Geriatrics 56 466-471

25- Rogers RS, Dawson AW, Wang Z, Thyfault JP, and Hinton PS. Acute response of serum markers of bone turnover to a single-bout of resistance-training or plyometrics. Journal of applied physiology. 2011 (1).

26- Saunders, M.M., Taylor, A.F., Du, C., Zhou, Z., Pellegrini Jr., V.D., Donahue, H.J., 2006. Mechanical stimulation effects on functional end effectors in osteoblastic MG-63 cells. J. Biomech. 39, 1419-1427.
27- Sherk VD, Chrisman C, Smith J, Young KC, Singh H, Bemben MG. Acute bone marker responses to whole-body vibration and resistance exercise in young women. J Clin Densitom. 2013;16(1):104-9.

28- Takeshima N, Rogers ME, Watanabe E, Brechue WF, Okada A, Yamada T, Islam MM, and Hayano J.: Water-based exercise improves healthrelated aspects of fitness in older women Medicine \&Amp Science In Sports \&Amp Exercise, 24: 544 $551 \cdot(2002)$

29- Tormen M. (2007). Effect of training and non training of a aquatic exercise program on profile lipids and in the bone reabsorption in pre-menopausal women [Master Dissertation]. Federal University of Rio Grande do Sul/Brazil.

30- Virtanen, P., Viitasalo, J. T., Vuori, J., Vaananen, K. \& Takala, T. E. (1993). Effect of concentric exercise on serum muscle and collagen markers. Journal of Applied Physiology 75, $1272-1277$.

31- Young A, Dinan S. (2005): Activity in later life BMJ 2005;330:189-91

32- Zakia AF. Sports training physiology (Book). Dar El Fekr El Araby, Cairo, Egypt (Ed.),p. 198, 2004 
